\title{
Cataract Detection
}

\author{
Mayur R Yadav ${ }^{1}$, Prof. N. M. Wagdarikar ${ }^{2}$ \\ PG Student, Department of E\&TC Engineering, Smt. Kashibai Navale College of Engineering, Pune India ${ }^{1}$ \\ Asst. Professor, Department of E\&TC Engineering, Smt. Kashibai Navale College of Engineering, Pune India ${ }^{2}$
}

\begin{abstract}
The percentage of blindness in today's world above the age of 65 is $78 \%$ out of which more than $55 \%$ of people are blind because they are suffering from cataract. The lens in the human eye enables one to see. It is made up of water and some proteins. It is normally transparent. But when a person is suffering from cataract, the lens losses its transparency and is formed when the protein clumps together and clouds the lens due to which the lens loses its transparency and the person is not able to see as the light cannot reach the eye. Cataract is the leading cause of blindness all over the world especially in senior citizens. Its initial symptoms are watering of eyes, blurred view, white spot can be seen in the eye.
\end{abstract}

Keywords: cataract, immature, mature, hypermature.

\section{INTRODUCTION}

Cataract is a kind of eye disease that is a clouding in the lens of the eye that affects vision. Cataract exhibits a lot of whitish colour inside a pupil. The three classes of cataracts are: normal, immature, mature and hypermature, which differ in seriousness. In an immature cataract, a whitish colour appears inside the pupil but less so than in mature or hypermature cataracts. Usually, the condition is not yet serious. A Hypermature cataract exhibits much whitish colour inside the pupil and can cause the lens of the eye to break if surgery is not carried out. This condition is very dangerous. The World Health Report published in 2001 estimated that there were 20 million people who are bilaterally blind (i.e., with eyesight of less than 3/60 in the better eye) whose blindness was caused by age related cataracts. That number will have increased to 40 million by the year 2020. Increasing age is associated with an increasing prevalence of cataracts, but in most developing countries, cataracts often occur earlier in life. One of the developing countries that have the highest number of people with cataracts is Indonesia. There are about 6 million people in Indonesia who suffer from cataracts, but Indonesia only has about 1160 ophthalmologists for a population of more than 200 million people (one for every 350.000 people). In addition, ophthalmologists are not evenly distributed. Many ophthalmologists are located in the capital city, yet many people have no access to ophthalmologists because of geographic conditions. This project will help people detect cataract even without the help of ophthalmologists.

\section{METHODOLOGY}

In this method, we extracted all information about cataract from pupil area only because all information about cataracts comes from the lens only. This is based on the fact that the opacities as an important sign of cataracts occur in the lens. However, when using a compact digital camera, we found problems such as insufficient image quality and uncontrolled illumination. For example, if we employ intensity value for screening cataract, i.e. higher intensity corresponds to a serious condition; it would fail for a cataract eye image taken under low illumination.

It appears that a non-serious condition eye image has an average intensity about 155 inside a pupil while a serious condition eye image only has an average intensity about 55 inside a pupil. In order to develop a robust cataract screening techniques, we proposed to use specular reflection analysis as the core method for cataract screening because specular reflection always brighter than surrounding area and it is not depend on illumination condition. We also were considering texture information as the supporting method.

\section{PRIOR APPROACH}

Utilization of Portable Digital Camera for Detecting Cataract-A paper by Retno Supriyanti, Hitoshi Habe and Masatsugu Kidode published by INTECH open science open minds, is based on step by step instructions on the use of digital cameras as the main equipment for detecting cataract, complete with an explanation of image processing techniques for the analysis of digital images produced by digital cameras. Regarding the conditions in developing countries which have limitations both of eye doctors and health facilities, using simple equipment such as digital camera for cataract screening is promising and sufficient. Because digital camera is small and easily carried out, easy to use and inexpensive. Also, the method for supporting digital camera has a good performance for distinguishing between serious and non-serious condition; therefore, it is very useful for determining people who need a surgery as 
soon as possible.

CATRA: Interactive Measuring and Modelling of Cataracts- A paper by Vitor F. Pamplona, Erick B. Passos, Jan Zizka, Manuel M. Oliveira, Everett Lawson, Esteban Clua, Ramesh Raskar and published by MIT Media Lab, UFRGS Instituto de Inform'atica, UFF Media Lab, Comenius University DAI is based on method to assess cataracts in the human eye by crafting an optical solution that measures the perceptual impact of forward scattering on the foveal region. Current solutions rely on highly-trained clinicians to check the back scattering in the crystallin lens and test their predictions on visual acuity tests. Close-range parallax barriers create collimated beams of light to scan through sub-apertures, scattering light as it strikes a cataract. User feedback generates maps for opacity, attenuation, contrast and sub-aperture point-spread functions. The goal is to allow a general audience to operate a portable high-contrast light-field display to gain a meaningful understanding of their own visual conditions. User evaluations and validation with modified camera optics are performed. Compiled data is used to reconstruct the individual's cataract-affected view, offering a novel approach for capturing information for screening, diagnostic, and clinical analysis.

Extracting Appearance Information inside the Pupil for Cataract Screening A paper by Retno Supriyanti, Hitoshi Habe, Masatsugu Kidode Graduate School of Information Science, Nara Institute of Science and Technology Japan (retnos; habe;kidode)@is.naist.jp published by Satoru Nagata Dept. Of Medical and Biomedical Engineering, Shiga Medical University Japan nagata@belle.shiga-med.ac.jp is based on method for cataract screening. Referring to the fact of increasing number of cataract especially for the people who lived in the developing countries, this kind of discussion will be important. The method used three kinds of features; specular reflection appearance, texture uniformity and average intensity inside the pupil. Actually, The core of this method is based on a specular reflection appearance inside the pupil. Referring to the reflection theorem, normal eye images will have two kinds of reflection inside the pupil while cataract eye images have one reflection and both of them are always in a line so we can easily distinguish between cataract and normal eye images. The result shows that percentage value of True Positive is $91.97 \%$ and percentage value of False Positive is $18.75 \%$. This performance is good enough for cataract screening especially in the developing countries

\section{OUR APPROACH}

The sample images that we have used in our project have been shown in the features. Block diagram is the graphical representation of our project. It shows the flow of the project what comes after a block and how the output is obtained

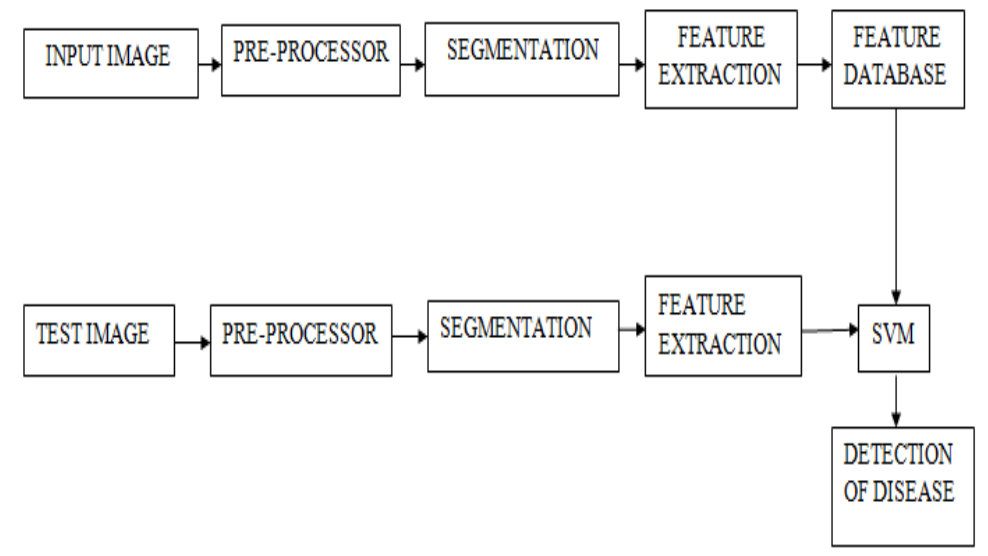

\section{INPUT IMAGE-}

We have distributed the images into four classes:

A] $0-10 \%$ (almost negligible) infected image of lens

B] $10-40 \%$ infected image of the lens

C] $40-70 \%$ infected image of the lens

D] $70-100 \%$ infected image of the lens

\section{TEST IMAGE-}

In this block the images of infected lens will be given as input and will be further given to the next block for testing

\section{PRE-PROCESSING-}

In this block three types of processes are done so that the image is enhanced-

A] The resizing of all the images is done so that the matrix of all the images is of equal dimension. This is done so that operations performed on the matrix are carried out successfully. 
B] The contrast enhancement of the region of interest is done so that it is clearly visible as compared to the region of non-interest and operations on the region of interest are carried out successfully.

C] Illumination distribution i.e. light adjustment of the image so that the region of non-interest is blurred and the shadows are removed from the image and the area of region of interest is calculated properly

\section{SEGMENTATION-}

Otsu's thresholding method is the best and simplest method to perform thresholding so we are using that method.

There are two regions in segmentation namely:

A] Foreground: The region of interest is called as the foreground and is highlighted.

B] Background: The region of non-interest is called as the background and is blurred.

Otsu's Thresholding-

In this method the image is converted from grey to binary image.

- The grey image consists of 256 values $(0-255)$ from which it selects one value that is the threshold value.

- All the pixels of the grey image are converted into binary pixels that is it consists of only two values 0 and 1 .

- All the values below the threshold value are converted into pixel value 0 that is they are white in color.

- All the values above the threshold value are converted into pixel value 1 that is they are black in color.

\section{FEATURE EXTRACTION-}

There are many ways in which we can detect the features of an image. Eigen value detection is used to detect shape, high frequency and colour. Random transform and random projection are used for invariant to direction. Gabor filter is used to detect texture features. DWT (Discrete Wavelet Transform) is used for edge and shape detection. Curvelet is used for point, curves and edge detection. Out of all these methods we are using curvelet and Gabor filter for feature extraction as they are the most simple and useful methods for our project.

6. SUPPORT VECTOR MACHINE (SVM)-

- SVM stands for Support Vector Machine.

- It separates the images into different classes such that all the images of one group are in the same class.

- It has the best separating line.

- When a test image is given it compares the test image with all the images that are there in the database and finds the closest distance of the test image from the input image.

- When the number of training data is small, SVMs outperform conventional classifiers.

- By maximizing margins performance of conventional classifiers can be improved.

- Formulated for two-class classification problems

- Map the input space into the feature space

- Determine the optimal hyperplane in the feature space

\section{FEATURE DATABASE-}

- The processes that are carried out in the pre-processing block, segmentation block and the feature extraction block give rise to the final image called the feature database.

- All these images are stored in the feature database block and are compared in SVM.

- These processes are been carried out on the input image as well as the test image and then the comparison of these images is done in SVM.

- The output of all these blocks is given to the SVM where the input image and the test image are compared.

8. DETECTION OF DISEASE-

- This is the last block of the block diagram where we get the final output.

- In this block will get to know whether a person is Suffering from cataract or not, if yes then by how Much severity.

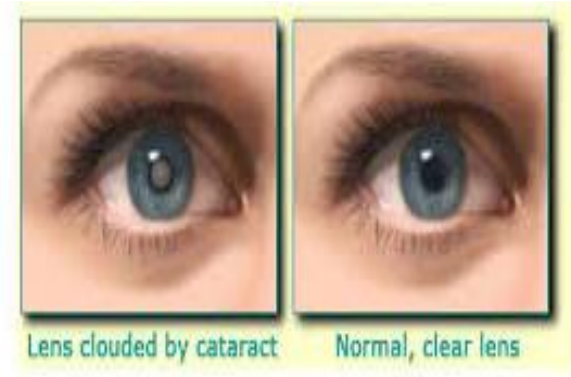




\section{CONCLUSION}

Cataracts are a common cause of decreased vision, particularly for the elderly, but they are treatable. Your ophthalmologist can tell you whether cataract or some other problem is the cause of your vision loss and can help you decide if cataract surgery is appropriate for you. Hence, in this project we have learned how cataract can be detected and treated at a low level. So that the rate of blindness over the world can be reduced. In this project we are trying to reduce the percentage of blindness from the world as it is the leading cause of blindness all over the world. It can be treated easily if detected at an early stage. Operation of the patient can be done with simplicity and less complications if detected at an early stage. The efficiency as calculated by us is $85 \%$.

\section{REFERENCES}

[1] Utilization of Portable Digital Camera for Detecting Cataract- A paper by Retno Supriyanti, Hitoshi Habe and Masatsugu Kidode Published by INTECH

[2] CATRA: Interactive Measuring and Modelling of Cataracts A paper by Vitor F. Pamplona, Erick B. Passos, Jan Zizka, Manuel M. Oliveira, Everett Lawson, Esteban Clua, Ramesh Raskar and published by MIT Media Lab, UFRGS Instituto de Inform’atica, UFF Media Lab, Comenius University DAI

[3] Extracting Appearance Information inside the Pupil for Cataract Screening A paper by Retno Supriyanti, Hitoshi Habe, Masatsugu Kidode Graduate School of Information Science, Nara Institute of Science and Technology Japan (retno-s;habe;kidode)@is.naist.jp published by Satoru Nagata Dept.of Medical and Biomedical Engineering, Shiga Medical University Japan nagata@ belle.shiga-med.ac.jp

[4] Automatic opacity detection in retro-illumination images for cortical cataract diagnosis by Huiqi Lia, Liling Koa,b, Joo Hwee Lima, Jiang Liua, Damon Wing Kee Wonga, Tien Yin Wongc, Ying Sunb. 